\title{
Serum Myoglobin Potentiates Acute Kidney Injury Following Exertional Heatstroke and Its Mechanisms Involved Endoplasmic Reticulum Stress-Associated Ferroptosis
}

\section{Ying-yi Luan}

Capital Medical University Beijing Obstetrics and Gynecology Hospital

\section{En-ping Huang}

Southern Medical University

\section{Rong-ping Zhou}

Nanchang University Medical College: Medical College of Nanchang University

Jia-jia Huang

Shenzhen Second People's Hospital

\section{Zhen-jia Yang}

Shenzhen Second People's Hospital

\section{Zhi-peng Zhou}

Shenzhen Second People's Hospital

Jingjing Zhang

Shenzhen Second People's Hospital

Yan Liu

Shenzhen Second People's Hospital

\section{Zhi-feng Liu}

General Hospital of Guangzhou Military Command of PLA: People's Liberation Army General Hospital of Southern Theatre Command

Ming Wu ( $\sim$ boshiyy@126.com )

Shenzhen Second People's Hospital https://orcid.org/0000-0001-9427-8211

\section{Research}

Keywords: Myoglobin, Exertional heatstroke, Acute kidney injury, Ferroptosis, Endoplasmic reticulum stress

Posted Date: October 29th, 2021

DOI: https://doi.org/10.21203/rs.3.rs-956335/v1 
License: (c) (i) This work is licensed under a Creative Commons Attribution 4.0 International License. Read Full License 


\section{Abstract}

Background: Myoglobin released by rhabdomyolysis (RM) is considered to be involved in the pathogenesis of kidney disease caused by crush injury, but whether a high level of serum myoglobin predisposes patients to acute kidney injury (AKI) and increases mortality following exertional heatstroke (EHS) and its molecular mechanisms are still unclear.

Methods: Serum myoglobin concentrations in patients with EHS were measured at admission, $24 \mathrm{~h}$ and $48 \mathrm{~h}$ after admission and discharge. The risk of AKI at 48 hours was the primary outcome, AKI at discharge and death at 90 days were the secondary outcome. In experimental studies, we further investigated the mechanisms of human kidney proximal tubular (HK-2) cells that were exposed to human myoglobin under heat stress conditions and the effect of baicalein.

Results: The myoglobin levels were assessed in 187 patients who were undergoing EHS, 82 who were undergoing AKI. The highest myoglobin quartile ( $v s$. the lowest) had an adjusted odds ratio (OR) of 18.95 ( $95 \%$ confidence interval [Cl], 6.00 to 59.83) for the primary outcome and the OR (vs. quartile 2) was 7.92 ( $95 \% \mathrm{Cl}, 1.62$ to 38.89 ) for the secondary outcome. The survival rate of HK-2 cells treated with myoglobin under heat stress was significantly decreased, and the production of $\mathrm{Fe}^{2+}$ and ROS was markedly increased, accompanied by changes in ferroptosis proteins, including increased p53, decreased SLC7A11 and GPX4, and alterations in ERS marker proteins. Treatment with baicalein attenuated HK-2 cell ferroptosis induced by myoglobin under heat stress through inhibition of ERS.

Conclusions: High serum myoglobin levels were associated with AKI and mortality following EHS, which mechanisms involved ferroptosis and ERS. Baicalein-targeted ERS- ferroptosis may be a potential therapeutic drug for the treatment of AKI in patients with RM after EHS.

\section{In Brief}

Excessive ofserum myoglobin released by rhabdomyolysis, involving in the pathogenesis of acute kidney injury (AKI) following exertional heatstroke (EHS), triggers ferroptosis and endoplasmic reticulum stress (ERS). Baicalein-targeted ERS associated ferroptosis may be a potential therapeutic drug for AKI induced by rhabdomyolysis after EHS.

\section{Highlights}

- Peak serum myoglobin concentrations predispose patients to acute kidney injury and increased mortality following exertional heatstroke.

- Taking myoglobin $\geq 1000 \mathrm{ng} / \mathrm{ml}$ as the serological diagnostic standard of rhabdomyolysis can more accurately predict the occurrence and 90-day prognosis of acute kidney injury in exertional heatstroke. 
- Excessive myoglobin may be a pathogenic factor in human kidney proximal tubular cells after heat stress by aggravating cell ferroptosis and the ERS response.

- Baicalein could be used as a potential therapeutic drug for rhabdomyolysis complicated acute kidney injury following exertional heatstroke.

\section{Introduction}

With global warming, a high-temperature climate has a disastrous impact on public health. Heatstroke is a clinical syndrome characterized by an imbalance in body temperature regulation, disorder of water and electrolytes, and impairment of nervous system function. Exertional heatstroke (EHS) has a poorer prognosis than classical heatstroke, which often combines rhabdomyolysis(RM) [1], acute kidney injury (AKI) [2], acute hepatic injury (AHI) [3], diffuse intravascular coagulation (DIC) [4], and even multiple organ dysfunction syndrome (MODS), with $40-70 \%$ mortality and $30 \%$ disability $[5,6]$.

Previous studies found that under high temperature and humidity conditions, vigorous exercise might lead to energy metabolism disorders of skeletal muscle and RM, and then intracellular myoglobin enters the circulation with blood, accompanied by free radicals, creatine kinase, mitochondrial DNA, histones, microRNAs and other substances, which could activate renal vascular endothelial cells or renal tubular epithelial cells [7-11]. Simultaneously, abundant free radicals mediate lipid peroxidation of the cell membrane and maladjusted inflammatory reactions, which can damage the capillary network adjacent to striated muscle cells, increase vascular permeability and further aggravate the oedema of striated muscle [12-14]. When skeletal muscle is injured, a large amount of myoglobin is released, which reaches the distal renal tubule through glomerular filtration and combines with urinary protein to form a renal tubular cast depending on the concentration of myoglobin in the initial urine, which eventually leads to an increase in renal intracapsular pressure and a decrease in the glomerular filtration rate[15-17]. On the other hand, myoglobin can also inhibit the synthesis of nitric oxide and lead to renal ischaemia, promoting the occurrence of AKI. Despite recent gains in our understanding of the causes and underlying mechanisms of RM complicated with AKI in heatstroke, few therapeutic or preventive options exist [1821]. Thus, uncovering new therapeutic targets for the prevention of RM complicated with AKI in heatstroke is important.

Inflammation and oxidative stress are central components of the pathogenesis of AKI, implicating multiple subtypes of immune cells. It is considered that the intracellular substances and type components released by RM are involved in the process of AKI, especially myoglobin jamming renal tubules and increasing pressure in renal vesicles [22-24]. During RM, glomerular filtered myoglobin enters the proximal renal small lumen. Through the endocytosis of renal tubular cells, myoglobin is pumped into the lysosome of tubular cells and decomposed into globulin and haeme, which are further decomposed into haem and iron ions [25-27]. If too much myoglobin is filtered, proximal renal tubular cells are persistently stimulated, which will eventually lead to iron ion overload in renal tubular cells; catalyse the peroxidation of phospholipids, protein or DNA; and finally lead to acute tubular necrosis or renal failure. Recent studies have shown that the direct injury of lipid peroxidation in proximal tubules 
induced by divalent iron ions produced by myoglobin metabolism through the Fenton reaction may be a cardinal mechanism of kidney injury induced by RM [28], in which ferritin heavy chain plays an important role in acute renal tubular injury. In the ferroptosis model induced by RM, various small molecule inhibitors (ferrostatin-1 and ferrostatin) to prevent lipid peroxidation could effectively inhibit cell necrosis induced by hydroxyquinoline and ammonium ferrous sulfate [29]. It is unclear whether myoglobin has an effect on ferroptosis in kidney tubular cells., i.e., the cells most affected in heatstroke complicated with AKI.

In the present study, the clinical and prognostic data of EHS patients admitted to the intensive care unit (ICU) in China over a 10-year period were retrospectively collected. We investigated whether a high level of myoglobin was associated with AKI in patients undergoing EHS and whether myoglobin could more accurately predict the occurrence and 90-day prognosis of AKI after EHS. We then used experimental models to determine the key roles of myoglobin in HK-2 cell survival during heat stress and revealed a previously unknown role and potential mechanism for myoglobin.

\section{Methods}

\section{Study design and participants}

A retrospective cohort study was performed in the ICU of the General Hospital of Southern Theatre Command of Peoples Liberation Army from January 2008 to June 2019. The inclusion criterion was as follows: patients with "exertional heatstroke" caused by strenuous exercise performed in a hightemperature and high-humidity environment. The diagnostic criteria of exertional heatstroke were as follows (1): exposure to a high temperature or high humidity and a history of strenuous exercise; a clinical syndrome causing an excessively high body temperature (central temperature higher than $40^{\circ} \mathrm{C}$ ); nervous system dysfunction (including delirium, cognitive impairment, coma, etc.); or systemic organ dysfunction. The exclusion criteria were as follows: 1) death or discharge within 24 hours after admission, 2) incomplete data regarding key indicators, 3 ) incomplete outcome evaluation data obtained via telephone follow-up, and 4) a previous history of organ dysfunction, such as chronic kidney disease. AKI was defined as KDIGO standard [30].

The cause of heatstroke is strenuous outdoor training in southern China, and all patients are treated with cooling immediately on site and continue to cool to $39^{\circ} \mathrm{C}$ (preferably $38.5^{\circ} \mathrm{C}$ to $38.0^{\circ} \mathrm{C}$ ) after admission. All patients received basic life support treatment according to their condition and were provided comprehensive treatment, including brain protection, anti-inflammation, administer fluids and organ function support, such as mechanical ventilation. Moreover, appropriate volume management was performed for patients with $\mathrm{AKI}$, and renal replacement therapy such as continuous renal replacement therapy (CRRT) was initiated if necessary.

\section{Heat Stress Cell Model}


HK-2 cells (National Colletion of Authenticated Cell Cultures, CBP60447) were cultured in Dulbecco's modified Eagle medium (DMEM, Lonza), and then HK-2 cells were treated with human myoglobin $(882.35 \mathrm{~nm} / \mathrm{l}$, cell assay concentration based on myoglobin renal threshold $1.5 \mathrm{mg} / \mathrm{dl}$ )(Abcam, Cambridge, MA, USA) at $43{ }^{\circ} \mathrm{C}$ for $2 \mathrm{~h}$ and rewarmed at $37^{\circ} \mathrm{C}$ for different time points $(0 \mathrm{~h}, 1 \mathrm{~h}, 3 \mathrm{~h}, 6 \mathrm{~h}$, and $12 \mathrm{~h})$. All cells are cultured at $37^{\circ} \mathrm{C}$ with $5 \% \mathrm{CO} 2(\mathrm{v} / \mathrm{v})$. All media were supplemented with $10 \%$ heat-inactivated foetal bovine serum, penicillin $(100 \mathrm{U} / \mathrm{ml})$, streptomycin $(100 \mathrm{U} / \mathrm{ml})$, and $1 \times$ GlutaMAX Supplement (Thermo Fisher Scientific).

\section{P53 Depletion}

Small interference RNA (siRNA) sequence that target p53 was designed by Shanghai GenePharma Co. Ltd (Shanghai, China), as shown below: siP53 (5'-CGAUGGUGUUACUUCCUGATT-3'). siRNA transfection was performed according to the instruction of Lipofectamine 3000 Transfection Reagent Kit from Invitrogen (Thermo Fisher Scientific). HK-2 cells seeded on a 6-well plate were grown to 70-90\% confluence. Lipofectamine 3000 reagent and $5 \mu \mathrm{M}$ siRNA or control siRNA were mixed with Opti-MEM (Thermo Fisher Scientific). The mixed solution was vortexed for 2-3s, and then incubated for $20 \mathrm{~min}$ at room temperature prior to incubation with cells for $6 \mathrm{~h}$. Thereafter, the siRNA/Lipofectamine 3000 complex medium was replaced with the same volume of regular fetal bovine serum-supplemented culture medium. Then, forty-eight hours after transfection, the depletion of P53 was confirmed by Western blotting, and cells were used in subsequent experiments.

\section{CCK8}

Human HK-2 cells were plated into 96 -well flat bottom plates at $2 \times 10^{5}$ cells/well, and incubated with myoglobin $(882.35 \mathrm{~nm} / \mathrm{I})$ and heat stress treatment in medium containing $10 \%$ foetal calf serum (FCS) at $37^{\circ} \mathrm{C}$ in $5 \% \mathrm{CO}_{2}$ in humidified air for 24 hours. Thereafter, $10 \mu$ CCK-8 solution (Dojindo, Japan) was added to each well, and the optical density of T-cell proliferative activity was measured by the use of a microplate reader.

\section{Western Blot}

Western blotting was performed to determine the expression of p-IRE1/IRE1, ATF-4, ATF-6, CHOP, P53, cystine glutamate transporter (SLC7A11) and glutathione peroxidase (GPX4) in HK-2 cells. Protein concentrations were measured using a bicinchoninic acid (BCA) protein assay kit (Thermo Scientific, Grand Island, NY, USA). Proteins of HK-2 cells were separated by SDS PAGE and then transferred electrophoretically onto polyvinylidenedifluoride (PVDF) membranes. The membranes were probed with anti-p-IRE1/IRE1 antibody, anti-ATF-4/6 antibody anti-CHOP antibody, anti-P53 antibody, anti-SLC7A11 antibody, anti-GPX4 antibody (Abcam, Cambridge, MA, USA) and polyclonal anti-actin antibodies (Santa Cruz Biotechnology, USA). Protein bands were detected using an Odyssey System from LI-COR Biosciences, USA. 


\section{Measurement of ROS and $\mathrm{Fe}^{2+}$}

The level of intracellular ROS was determined by the oxidation-sensitive fluorescent probe DCFH-DA with flow cytometry. The level of $\mathrm{Fe}^{2+}$ was detected by an iron assay kit purchased from Nanjing Jiancheng Bioengineering Institute and tested at a wavelength of $520 \mathrm{~nm}$.

\section{Statistical analysis.}

The continuous variables conforming to a normal distribution are expressed as $\bar{x} \pm s$. Count data and ordinal data are represented as medians and interquartile ranges (IQRs). Count data were compared using multiple independent samples nonparametric Kruskal-Wallis $\mathrm{H}$ tests, and measurement data for intergroup comparisons were analysed using nonparametric Mann-Whitney $\mathrm{U}$ tests. Datasets were examined by one-way ANOVA. The patient-related endpoints were the occurrence of AKI and AKI at discharge and 90-day mortality after admission. Statistical analyses were performed using SPSS Windows version 23.0 (SPSS Inc., Chicago, IL), Empower (R) (http://www.empowerstats.com, X\&Y solutions, Inc., Boston, MA) and R (http://www.R-project.org) software. P values (two-tailed) less than 0.05 were considered statistically significant. The study was approved by the Research Ethics Committee of the General Hospital of Southern Theatre Command of PLA (HE-2020-09). In view of the retrospective study design and depersonalization of data, the Ethics Committee agreed to waive the requirement for patient written informed consent but required that the patients be informed of the study details during a telephone follow-up.

\section{Results}

\section{Outcomes and baseline characteristics of serum biomarkers of RM in patients who underwent EHS} stratified according to the incidence of AKI

A total of 187 patients were included, and all of them were males. Of these, 82 (43.9\%) patients had AKI at admission, and stage 1, stage 2 and stage 3 were $62 / 82$ (75.6\%), 11/82 (13.4\%) and 9/82 (11.0\%), respectively. Twenty-one (12.4\%) patients had AKI at discharge, and 10/21 (47.6\%), 6/21 (28.6\%) and $5 / 21(23.8 \%)$ patients had stage 1, stage 2 and stage 3 AKI, respectively. The 90-day mortality was higher in AKI than in non-AKI $(26.8 \%$ vs. $1.0 \%, P<0.001)$. The levels of MB and CK at admission, 24 hours, 48 hours, and discharge were higher in patients with AKI than in non-AKI patients. (Table 1). 
Table 1

Clinical serum biomarker of rhabdomyolysis and outcomes of patients who underwent EHS, stratified according to incidence of acute kidney injury

\begin{tabular}{|c|c|c|c|}
\hline Variables & Non-AKI(n=105) & $\operatorname{AKI}(n=82)$ & $P$ value \\
\hline Age (years), Mean (SD) & $23.6 \pm 8.0$ & $26.1 \pm 11.0$ & 0.128 \\
\hline Males, N (\%) & $105(100 \%)$ & $82(100 \%)$ & 1.000 \\
\hline \multicolumn{4}{|l|}{ Outcomes N (\%) } \\
\hline AKI at admission & & $82 / 187(43.9 \%)$ & \\
\hline Stage 1 & & $62 / 82(75.6 \%)$ & \\
\hline Stage 2 & & $11 / 82(13.4 \%)$ & \\
\hline Stage 3 & & $9 / 82(11.0 \%)$ & \\
\hline AKI discharge & & $21 / 169(12.4 \%)$ & \\
\hline Stage 1 & & $10 / 21(47.6 \%)$ & \\
\hline Stage 2 & & $6 / 21(28.6 \%)$ & \\
\hline Stage 3 & & $5 / 21(23.8 \%)$ & \\
\hline 90-day mortality & $1 / 105(1.0 \%)$ & $22 / 82(26.8 \%)$ & $<0.001$ \\
\hline \multicolumn{4}{|l|}{ Serum biomarker of RM } \\
\hline \multicolumn{4}{|c|}{ MB (ng/ml), median (IQR) } \\
\hline admission & $193.6(63.2-583.5)$ & $1000.0(469.9-1000.0)$ & $<0.001$ \\
\hline 24 hours & $68.8(24.0-206.6)$ & $461.8(142.0-1000.0)$ & $<0.001$ \\
\hline 48 hours & $61.0(33.5-298.1)$ & $485.0(188.8-1000.0)$ & $<0.001$ \\
\hline Discharge & $38.5(21.0-76.5)$ & $516.0(90.0-1000.0)$ & $<0.001$ \\
\hline \multicolumn{4}{|l|}{ CK(U/L), median (IQR) } \\
\hline admission & $793.5(248.5-2148.2)$ & $1119.0(483.0-3518.0)$ & 0.028 \\
\hline 24 hours & $716.5(233.5-2531.8)$ & $2083.0(822.0-4768.5)$ & $<0.001$ \\
\hline 48 hours & $61.0(33.5-298.1)$ & $485.0(188.8-1000.0)$ & 0.005 \\
\hline Discharge & $38.5(21.0-76.5)$ & $516.0(90.0-1000.0)$ & 0.036 \\
\hline \multicolumn{4}{|c|}{ CK-Mb (ng/ml), median (IQR) } \\
\hline admission & $32.5(22.0-59.8)$ & $45.0(29.0-101.5)$ & 0.007 \\
\hline 24 hours & $32.5(17.2-62.0)$ & $57.0(32.0-104.5)$ & 0.003 \\
\hline
\end{tabular}




\begin{tabular}{|llll|}
\hline Variables & Non-AKI(n=105) & AKI $(\mathrm{n}=82)$ & $P$ value \\
\hline 48 hours & $28.0(21.0-60.0)$ & $40.0(21.0-73.0)$ & 0.284 \\
\hline Discharge & $13.0(8.8-19.0)$ & $16.0(7.7-30.5)$ & 0.214 \\
\hline
\end{tabular}

Association of Myoglobin and Acute Kidney Injury and 90-day Mortality Following Exertional Heatstroke

The characteristics of the patients, stratified according to quartiles of myoglobin level, are shown in Figure 1. For rhabdomyolysis after heatstroke, the incidence of AKI was $73.08 \%$ in the highest myoglobin quartile $(\geq 1000 \mathrm{ng} / \mathrm{ml})$ and $12.82 \%$ in the lowest quartile $(<127 \mathrm{ng} / \mathrm{ml})$, which yielded an adjusted odds ratio of AKI that was 18.95 (95\% confidence interval [Cl], 6.00-59.83) times as high in the highest quartile as in the lowest quartile (Fig. 1). The myoglobin level was also strongly associated with the combined outcome of acute kidney injury and death at 90 days (adjusted odds ratio, $7.9 ; 95 \% \mathrm{Cl}, 1.61$ to 38.89 ) (Fig. 2).

\section{Myoglobin is Associated with HK-2 Cell Ferroptosis in a Heat Stress Model}

To determine the role of myoglobin in the mechanism of human kidney proximal tubular (HK-2) cell function, $\mathrm{HK}-2$ cells were treated with myoglobin at concentrations of $882.35 \mathrm{~nm} / \mathrm{l}$ at $43^{\circ} \mathrm{C}$ for 2 hours and rewarmed at $37^{\circ} \mathrm{C}$ at different time points $(0 \mathrm{~h}, 1 \mathrm{~h}, 3 \mathrm{~h}, 6 \mathrm{~h}$, and $12 \mathrm{~h})$. First, we examined the effect of heat shock and myoglobin on the survival of $\mathrm{HK}-2$ cells, and the cell survival rate began to decline after $0 \mathrm{~h}$ of rewarming (Fig. 3A, $\mathrm{P}<0.01$ ). Simultaneously, with the extension of rewarming time, the cell survival rate decreased significantly; after $12 \mathrm{~h}$ of rewarming, the cell survival rate decreased to less than $50 \%$. We further determined whether ferroptosis was involved in severe heatstroke complicated with AKI. P53, SLC7A11 and GPX4 expression in HK-2 cells was detected by Western blot. We found that compared with the control group at $37^{\circ} \mathrm{C}$, the expression of p53 was significantly increased in response to myoglobin after heat stress for $0 \mathrm{~h}$. With the extension of rewarming time, the expression increased and then decreased and reached its highest level at $3 \mathrm{~h}$ of rewarming (Fig. 3B, P $<0.01$ ). As shown in Figure 1C, the expression of SLC7A11 and GPX4 was significantly decreased after heat stress and myoglobin treatment for $0 \mathrm{~h}$, and the expression continuously decreased with the extension of rewarming time. Furthermore, the change in intracellular iron content is a very important indicator of ferroptosis. We evaluated HK-2 cell ferroptosis by detecting the intracellular iron content and found that intracellular $\mathrm{Fe}^{2+}$ was markedly increased at $3 \mathrm{~h}$ of rewarming after heat stress and myoglobin treatment (Fig. $3 \mathrm{C}, \mathrm{P}<$ 0.01). Additionally, the level of ROS was significantly increased at $3 \mathrm{~h}$ of rewarming in the heat stressand myoglobin-treated cell model, suggesting that heat shock and myoglobin could induce the production of ROS and activate oxidative stress in HK-2 cells (Fig. 3D, P<0.01). Then, silencing of the P53 gene was used via an siRNA approach. SLC7A11 and GPX4 expression in HK-2 cells was significantly decreased at $3 \mathrm{~h}$ of rewarming in response to myoglobin under heat stress, and these effects were prevented by P53 gene silencing in HK-2 cells (Fig. 3E, P<0.01). Taken together, our data show that myoglobin appears to be related to human HK-2 cell ferroptosis under heat stress by affecting SLC7A11 and GPX4 expression, intracellular iron content, and the production of ROS. 
We further analysed the expression of ERS-related proteins by Western blot, mainly detected the three classical pathways of ERS, and analysed the expression of ATF-4, CHOP, ATF-6, IRE1 and p-IRE1. HK-2 cells treated with myoglobin and heat stress showed elevated expression of p-IRE1/IRE1, ATF-4, ATF-6 and $\mathrm{CHOP}$, and with the extension of rewarming time, their expression first increased and then decreased (Fig. 4A, $\mathrm{P}<0.01$ ). To confirm this, we used an ERS inhibitor (4-BPA) to treat $\mathrm{HK}-2$ cells. There was a significant increase in the expression of p-PER/PERK, p-eIF2, p-IRE1/IRE1, ATF-4, ATF-6 and CHOP at $3 \mathrm{~h}$ of rewarming in response to myoglobin under heat stress; thus, pretreatment with 4-BPA markedly decreased the expression of p-PER/PERK, p-elF2, p-IRE1/IRE1, ATF-4, ATF-6 and CHOP in the heat stress and myoglobin groups (Fig. 4B, $\mathrm{P}<0.01$ ). Together, these data reveal the important role of myoglobin in aggravating the ERS response in HK-2 cells during heatstroke.

\section{Myoglobin modulates HK-2 cell Ferroptosis involving Endoplasmic Reticulum Stress after Heat Stress}

ERS is closely related to ferroptosis. The above results suggest that both ERS and ferroptosis are involved in the process of heat stress- and myoglobin-mediated renal tubular injury, but the definite regulatory mechanism is not clear. To address whether myoglobin modulates ferroptosis by interacting with ERS, we performed 4-BPA to pull down ERS activation in HK-2 cells before heat stress and myoglobin treatment. We found that the cell survival rate was significantly decreased after heat stress and myoglobin treatment rewarming for $3 \mathrm{~h}$, and the cell survival rate was markedly increased in the 4-BPA + myoglobin+ heat stress group (Fig. 5A, P < 0.01). Western blot analysis showed that inhibition of ERS using 4-BPA decreased p53 activation and enhanced SLC7A11 and GPX4 expression in HK-2 cells after heat stress and myoglobin treatment (Fig. 5B, P < 0.01). Notably, intracellular Fe ${ }^{2+}$ and ROS were significantly decreased in response to heat stress and myoglobin combined with 4-BPA treatment rewarming for 3 hours (Fig. $5 \mathrm{C}$ and $\mathrm{D}, \mathrm{P}<0.01$ ), indicating that inhibiting ERS could suppress the accumulation of iron and ROS production induced by myoglobin under heat stress.

\section{Baicalein Protects HK-2 Cell Ferroptosis Induced by Myoglobin under Heat Stress by Inhibiting Endoplasmic Reticulum Stress}

Recent studies have reported that ERS and ferroptosis are involved in AKI induced by heat stress combined with myoglobin, and ERS inhibitors can effectively inhibit ferroptosis and alleviate heat stress and myoglobin-mediated kidney injury. Therefore, we considered whether there are clinical drugs that can inhibit ferroptosis by regulating ERS to alleviate rhabdomyolysis-induced AKI after EHS. Previous studies

have shown that baicalein can inhibit ferroptosis during tumours, sepsis and hypoxic renal injury [31-33], but its mechanism is not completely clear. In the present study, as expected, treatment with baicalein increased the cell survival rate after heat stress and myoglobin treatment rewarming for $3 \mathrm{~h}$ (Fig. $6 \mathrm{~A}, \mathrm{P}<$ 0.01 ), suggesting the protective role of baicalein in AKI under heat stress. Furthermore, baicalein inhibited the activation of ERS signalling, as demonstrated by decreased expression of $p-P E R / P E R K, p$-elF2, $p$ IRE1/IRE1, ATF-4, ATF- 6 and CHOP in the heat stress and myoglobin treatment rewarming for $3 \mathrm{~h}$ group 
(Fig. 6B, P < 0.01). Simultaneously, baicalein alleviated ferroptosis in HK-2 cells, as shown by decreased p53 activation and elevated SLC7A11 and GPX4 expression (Fig. 6C, P<0.01). Notably, intracellular Fe ${ }^{2+}$ and ROS were markedly decreased in response to heat stress and myoglobin combined with baicalein treatment rewarming for 3 hours (Fig. 6D and $E, P<0.01$ ), indicating that baicalein could inhibit the accumulation of iron and ROS production induced by myoglobin under heat stress.

\section{Discussion}

This retrospective cohort study showed that myoglobin was associated with subsequent AKI following EHS. Concurrently, we obtained experimental evidence that myoglobin might be directly involved in the pathogenesis of heat stress in HK-2 cells by modulating cellular ferroptosis and increasing endoplasmic reticulum stress. Inhibiting endoplasmic reticulum stress attenuated ferroptosis in HK-2 cells exposed to myoglobin under heat stress.

There has been little progress in the overall risk stratification, prevention, and treatment of AKI induced by RM after EHS. AKI is a potential complication of severe rhabdomyolysis, regardless of whether rhabdomyolysis is the result of trauma or some other cause, and the prognosis is substantially worse if renal failure develops. Long-term survival among patients with rhabdomyolysis and AKI is reported to be close to $80 \%$, and the majority of patients with RM-induced AKI recover renal function [34]. Myoglobin, a dark red 17.8-kDa protein, can transport and store oxygen in muscle cells [35]. However, cellular release of myoglobin leads to uncontrolled leakage of reactive oxygen species, and free radicals cause cellular injury [28]. More recently, it has been shown that myoglobin itself can exhibit peroxidase-like enzyme activity that leads to uncontrolled oxidation of biomolecules, lipid peroxidation, and the generation of isoprostanes [36]. We found that myoglobin levels were predictive of AKI after EHS, independent of relevant clinical characteristics, including baseline kidney function. In addition, the highest myoglobin quartile ( $v s$. the lowest, quartile 1) had an adjusted odds ratio of 18.95 (95\% confidence interval [Cl], 6.00 to 59.83) for $\mathrm{AKI}$, and the OR (vs. quartile 2) was $7.92(95 \% \mathrm{Cl}, 1.62$ to 38.89$)$ for AKI at discharge and death at 90 days. Improved assessment of the risk of AKI after EHS would allow for more informed decision-making and would help to identify a subgroup of patients who would benefit from an intervention to minimize AKI, potentially in the form of anti-myoglobin therapies.

The wide spectrum of clinical contexts in which myoglobin levels are associated with the incidence of EHS-complicated AKI. On the basis of our cell models, we speculate that there may be a synergistic effect between myoglobin, which may enhance the intracellular $\mathrm{Fe}^{2+}$ and ROS accumulation of human kidney proximal tubular cells, leading to ferroptosis. Ferroptosis is a new type of cell death that was discovered in recent years and is usually accompanied by a large amount of iron accumulation and lipid peroxidation during the cell death process; the occurrence of ferroptosis is iron-dependent [37, 38]. Ferroptosis-inducing factors can directly or indirectly affect glutathione peroxidase through different pathways, resulting in a decrease in antioxidant capacity and accumulation of lipid reactive oxygen species (ROS) in cells, ultimately leading to oxidative cell death $[39,40]$. Decades of evidence proved that ferroptosis is positively regulated by $p 53$, cysteinyl transferase ribonucleic acid synthase (CARS), 
transferrin receptor (TFR1), SLC7A11 and heat shock protein $\beta 1$ (HSP $\beta 1)$, nuclear factor E2 related factor 2 (NRF2), and GPX4[41]. Among the many members of the GPX family, GPX4 plays a pivotal role in the occurrence of ferroptosis and is the key regulator of its occurrence, mainly by inhibiting the formation of lipid peroxides [42]. P53 signalling has also been shown to downregulate the expression of SLC7A11 and affect the activity of GPX4, resulting in the reduction of antioxidant capacity, ROS accumulation, and ferroptosis [43-45]. Using a model of in vitro heat stress, we found that the expression of p53 was increased and the expression of SLC7A11 and GPX4 was markedly decreased, accompanied by a large amount of $\mathrm{Fe}^{2+}$ accumulation and ROS production, in response to myoglobin under heat stress in HK-2 cells. The absence of GPX4 can increase the accumulation of lipid peroxidation products in renal tubular epithelial cells, that is, the hydrogen peroxide lipid $\mathrm{L}-\mathrm{OOH}$, and eventually lead to acute renal failure [46]. We confirmed the occurrence of ferroptosis in HK-2 cells induced by myoglobin under heat stress. It is known that if the serum myoglobin is more than $100 \mathrm{mg} / \mathrm{dl}$, there will be tawny urine and apoptosis of renal tubular cells [47]. The concentration of myoglobin $(1.5 \mathrm{mg} / \mathrm{dl})$ is just the lowest value of myoglobinuria (renal threshold), indicating that myoglobin concentration has different effects on renal tubular injury, emphasizing the possibility of ferroptosis in the early stage of AKI.

Recent studies have shown that ERS plays an important role in the process of ferroptosis through the induction of unfolded proteins [48]. ERS, a common stress response in eukaryotic cells, is regulated by multiple factors, including the inositol requiring enzyme 1 (IRE1)-X-box binding protein 1 (XBP1)/TNF receptor associated factor 2 (TRAF2) pathway, ATF6 pathway and PERK-eukaryotic translation initiation factor-2 (elF2a)-ATF4 pathway $[49,50]$. During excessive ERS, unfolded or misfolded proteins continue to accumulate, resulting in the elevation of the expression of pro-apoptotic transcription factor DNA damage inducible transcription 3 (DDIT3, CHOP), which induces cell damage [51, 52]. ERS plays an important regulatory role in the occurrence and development of many diseases, such as primary glomerulopathy, diabetic nephropathy, drug-related tubulointerstitial injury, renal ischaemia-reperfusion injury, and many other kidney diseases. Our findings firmly establish that myoglobin aggravates the ERS response in HK-2 cells during heatstroke, as shown by the elevated expression of p-PER/PERK, p-eIF2, p-IRE1/IRE1, ATF-4, ATF-6 and CHOP. Of note, the ERS response is closely related to ferroptosis, whose agonist can synchronously activate the ERS response in cancer cells, and the activation of the ERS pathway inhibits ferroptosis and leads to drug resistance in cancer cells [53,54]. In some pathological conditions, the activation of the ERS pathway through the induction of unfolded proteins exacerbates the occurrence of ferroptosis $[55,56]$. Additionally, ferroptosis could promote the cystine-glutamate antiporter system Xc-, which leads to ERS $[57,58]$. Whether ERS is involved in the process of ferroptosis induced by myoglobin under heat stress is unknown. Therefore, the mechanism was verified in HK-2 cells in detail. In the in vitro experiments, it was demonstrated that attenuating ferroptosis by an ERS inhibitor could directly increase cell viability and GPX4 and SLC7A11 levels, decrease p53 activation, and decrease ROS and intracellular $\mathrm{Fe}^{2+}$ in response to myoglobin after heat stress, indicating that myoglobin modulated HK-2 cell ferroptosis involving the ERS response after heat stress. 
Although some progress has been made in the treatment of RM complicated with AKI after heatstroke, uncovering new therapeutic targets for the prevention of AKI following heatstroke complicated with RM is important. Based on the above observations, we further determined whether drugs associated with inhibition of ERS or ferroptosis would be useful for the treatment of AKI after heatstroke complicated with RM. Deferoxamine can inhibit lipid peroxidation, reduce the ferrous form of myoglobin, and alleviate RM and renal injury in rats, but it has a certain toxicity to the kidney due to the hydrophilicity of deferoxamine. Deferoxamine oral substitutes for a-hydroxypyridines, such as desferridone, have been confirmed by a large number of clinical trials and can be used in patients with high ferrous load after repeated blood transfusion. However, no studies have shown that deferrone can effectively prevent or treat AKI caused by RM. Baicalein is a plant-derived flavonoid that has antioxidant, antiapoptotic and anti-inflammatory effects in a variety of diseases. Through the multidimensional multi-information (md-mi) xanthine oxidase and superoxide anion fingerprint, baicalein mainly inhibits xanthine oxidase (XOD), and baicalein produced in Gansu Province in China has the best activity in both inhibiting XOD activity and scavenging reactive oxygen species and regulating different cell deaths by inhibiting reactive oxygen species [59]. Baicalein can promote the functional recovery of spinal cord ischaemia-reperfusion injury by inhibiting focal death, reducing ERS-mediated apoptosis and activating autophagy. Baicalein can also block arachidonic acid 12 lipoxygenase (ALOX12), which is necessary for p53 to mediate tumour inhibition through different ferroptosis pathways $[60,61]$. This experiment found that baicalein played a vital role in the process of myoglobin-mediated renal tubular injury after heat stress and effectively relieved myoglobin-mediated renal tubular injury by inhibiting ERS and ferroptosis.

\section{Conclusions}

In summary, serum myoglobin concentrations were associated with incident AKI induced by RM after EHS. Taking MB $\geq 1000 \mathrm{ng} / \mathrm{ml}$ as the biomarker of RM can more accurately predict the occurrence and 90 day prognosis of AKI in EHS. The experimental models used here suggest that excessive myoglobin may be a pathogenic factor in human kidney proximal tubular cells after heat stress by aggravating cell ferroptosis and the ERS response. Baicalein might be a targeted therapeutic drug to reduce acute kidney injury associated with myoglobin under EHS.

\section{Declarations}

\section{Acknowledgements}

Not applicable.

\section{Authors' contributions}

All authors had full access to all the data in the study and take responsibility for the integrity of the data and the accuracy of the data analysis. Zhifeng Liu and Ming Wu were responsible for study concept and design. Ming Wu and Zhifeng Liu were responsible for collecting the data. En-ping Huan, Rong-ping 
Zhou and Yan Liu were responsible for statistical analysis. Ying-yi Luan and Ming Wu were responsible for drafting the manuscript.

\section{Funding}

This work was supported by grants from the National Natural Science Foundation of China [N0.82072143], Sanming Project of Medicine in Shenzhen (SZSM20162011), Shenzhen Science and Technology Innovation Commission (JCYJ20190806163603504), and Shenzhen Second People's Hospital Clinical Research Fund of Guangdong Province High-level Hospital Construction Project (Grant No20173357201815, No20193357003, No 20203357014).

\section{Availability of data and materials}

All data generated or analysed during this study are included in this published article and its supplementary files.

\section{Ethics approval and consent to participate}

The study was approved by the Research Ethics Committee of the General Hospital of Southern Theatre Command of PLA (HE-2020-09). In view of the retrospective study design and depersonalization of data, the Ethics Committee agreed to waive the requirement for patient written informed consent but required that the patients be informed of the study details during a telephone follow-up.

\section{Consent for publication}

Not applicable.

\section{Competing interests}

The authors declare that they have no competing interests.

\section{References}

1. Wu M, Wang CL, Liu ZY, et al. SOFA Score for Prediction of Mortality of Patients With Rhabdomyolysis Following Exertional Heatstroke: A Longitudinal Cohort Study in Southern China. Front Med (Lausanne). 2021;9:724319.

2. Wu M, Wang Z, Liu ZY, et al. Clinical characteristics and risk factors associated with acute kidney injury inpatient with exertional heatstroke: an over 10-year intensive care survey. Front Med (Lausanne). 2021;8:678434.

3. Ji J, Gao J, Wang C, Ouyang L,et al. Characteristics and outcome of exertional heatstroke patients complicated by acute hepatic injury: A cohort study. J Clin Transl Hepatol. 2021;00(00):00-0. doi: 
10.14218/ JCTH.2021.00084. [Epub ahead of print].

4. Zhong L, Wu M, Wang C, et al. Clinical characteristics and outcomes of patients with severe heatstroke complicated with disseminated intravascular coagulation: A case-control study. Thromb Res. 2021;197:120-23.

5. ilma B, Derhaschnig U. Disseminated intravascular coagulation in heat stroke: A hot topic. Crit Care Med. 2012;40(4):1370-2.

6. Epstein Y, Yanovich R. Heatstroke N Engl J Med. 2019;380(25):2449-59.

7. Moreno JA, Manin-Cleary C, Gutrez E, et al. AKI associated with macroscopic glomerular hematuria: clinical and pathophysiologic consequences. Clin JAmSocNephml. 2012;7(1):175-84.

8. Jang HR, Rabb H. The innate immune response in ischemic acute kidney injury. clin Immunol. 2009;130(1):4I-50.

9. Gonzalez-Michaca L, Farrugia G, Croatt AJ, et al. Heme: a deteminallt of life a11d death in renal tubular epithelial cells. Am J Physiol Renal Physiol. 2004;286(2):F370-7.

10. Moreno JA, Marlin Cleary C, Guti6rrez E. et al. Haematua: the forgotten CKD factor. Nephrol Dial Transplant. 2012;27(1):28-34.

11. Wagener FA, Feldman E, de Witte T, et al. Heme induces the expression of adhesion molecules ICAM1,VCAM-1, and E selectinin vascular endothelial cells. Proc Soc Exp BiolMed. 1997;216(3):456-63.

12. Giannoglou GD, Chatzizisis YS, Misirli G. The syndrome of rhabdomyolysis: Pathophysiology and diagnosis. Eur J Intern Med. 2007;18(2):90-100.

13. Guis S, Mattei JP, Cozzone PJ, et al. Pathophysiology and clinical presentations of rhabdomyolysis. Joint Bone Spine. 2005;72(5):382-91.

14. Komada T, Usui F, Kawashima A, et al. Role of NLRP3 Inflarllmasomes for Rhabdomyo1ysis-induced Acute Kidney Injury. Sci Rep. 2015;5:10901.

15. Ng JH, Bijol V, Sparks MA, et al. Pathophysiology and Pathology of Acute Kidney Injury in Patients With COVID-19. Adv Chronic Kidney Dis. 2020;27(5):365-76.

16. Petejova N, Martinek A. Acute kidney injury due to rhabdomyolysis and renal replacement therapy: a critical review. Crit Care. 2014;18(3):224.

17. Okubo K, Kurosawa M, Kamiya M, et al. Macrophage extracellular trap formation promoted by platelet activation is a key mediator of rhabdomyolysis-induced acute kidney injury. Nat Med. 2018;24(2):232-8.

18. Grivei A, Giuliani KTK, Wang X, et al. Oxidative stress and inflammasome activation in human rhabdomyolysis-induced acute kidney injury. Free Radic Biol Med. 2020;160:690-5.

19. Shanu A, Groebler L, Kim HB, et al. Selenium inhibits renal oxidation and inflammation but not acute kidney injury in an animal model of rhabdomyolysis. Antioxid Redox Signal. 2013;18(7):756-69.

20. Scharf C, Liebchen U, Paal M, et al. Blood purification with a cytokine adsorber for the elimination of myoglobin in critically ill patients with severe rhabdomyolysis. Crit Care. 2021;25(1):41. 
21. Weidhase L, de Fallois J, Haußig E, et al. Myoglobin clearance with continuous veno-venous hemodialysis using high cutoff dialyzer versus continuous veno-venous hemodiafiltration using high-flux dialyzer: a prospective randomized controlled trial. Crit Care. 2020;24(1):644.

22. Kamal F, Snook L, Saikumar JH. Rhabdomyolysis-Associated Acute Kidney Injury With Normal Creatine Phosphokinase. Am J Med Sci. 2018;355(1):84-7.

23. Panizo N, Rubio-Navarro A, Amaro-Villalobos JM, et al. Molecular Mechanisms and Novel Therapeutic Approaches to Rhabdomyolysis-Induced Acute Kidney Injury. Kidney Blood Press Res. 2015;40(5):520-32.

24. Messerer DAC, Halbgebauer R, Nilsson B, et al. Immunopathophysiology of trauma-related acute kidney injury. Nat Rev Nephrol. 2021;17(2):91-111.

25. Zorova LD, Pevzner IB, Chupyrkina AA, et al. The role of myoglobin degradation in nephrotoxicity after rhabdomyolysis. Chem Biol Interact. 2016;256:64-70.

26. Guerrero-Hue M, García-Caballero C, Palomino-Antolín A, et al. Curcumin reduces renal damage associated with rhabdomyolysis by decreasing ferroptosis-mediated cell death. FASEB J. 2019;33(8):8961-75.

27. Geng Y, Ma Q, Du YS, et al. Rhabdomyolysis is Associated with In-Hospital Mortality in Patients with COVID-19. Shock. 2021; 56(3):360-367.

28. Bosch X, Poch E, Grau JM. Rhabdomyolysis and acute kidneyinjury. N Engl J Med. 2009;361(1):6272.

29. Skouta R, Dixon SJ, Wang J, et al. Ferrostatins inhibit oxidative lipiddamage and cell death in diverse disease models. J Am Chem Soc. 2014;136(12):4551-6.

30. Stevens PE, Levin A. Kidney Disease: Improving Global Outcomes Chronic Kidney Disease Guideline Development Work Group M. Evaluation and management of chronic kidney disease: synopsis of the kidney disease: improving global outcomes 2012 clinical practice guideline. Ann Intern Med. 2013;158:825-30.

31. Elmarakby AA, Ibrahim AS, Katary MA, et al. A dual role of 12/15-lipoxygenase in LPS-induced acute renal inflammation and injury. BiochimBiophys Acta Mol Cell Biol Lipids. 2019;186411(11):1669-80.

32. Wu K, Li H, Tian J, et al. Protective effect of baicalein on renal ischemia/reperfusion injury in the rat. Ren Fail. 2015;372(2):285-91.

33. Wang $\mathrm{M}$, Dong $\mathrm{Y}, \mathrm{Wu} \mathrm{J}$, et al. Baicalein ameliorates ionizing radiation-induced injuries by rebalancing gut microbiota and inhibiting apoptosis. Life Sci. 2020;261:118463.

34. Woodrow G, Brownjohn AM, Turney JH. The clinical and biochemical features of acute renal failure due to rhabdomyolysis. Ren Fail. 1995;17:467-74.

35. Knochel JP. Rhabdomyolysis and myoglobinuria. Annu Rev Med. 1982;33:435-43.

36. Reeder BJ, Wilson MT. Hemoglobin and myoglobin associated oxidative stress: from molecular mechanisms to disease states. Curr Med Chem. 2005;12:2741-51. 
37. Dixon SJ, Lemberg KM, Lamprecht MR, et al. Ferroptosis: an iron-dependent form of nonapoptotic cell death. Cell. 2012;149(5):1060-72.

38. Xie Y, Hou W, Song X, et al. Ferroptosis: process and function. Cell Death Differ. 2016;23(3):369-79.

39. Friedmann Angeli JP, Schneider M, Proneth B, et al. Inactivation of the ferroptosis regulator Gpx4 triggers acute renal failure in mice. Nat Cell Biol. 2014;16(12):1180-91.

40. Li J, Cao F, Yin HL, et al. Ferroptosis: past, present and future. Cell Death Dis. 2020;11(2):88.

41. Wang Y, Kuramitsu Y, Baron B, et al. PERK/CHOP contributes to the CGK733-induced vesicular calcium sequestration which is accompanied by non-apoptotic cell death. Oncotarget. 2015;6(28):25252-65.

42. Rui T, Li Q, Song S, et al. Ferroptosis-relevant mechanisms and biomarkers for therapeutic interventions in traumatic brain injury. Histol Histopathol. 2020;35(10):1105-13.

43. iang L, Kon N, Li T, et al. Ferroptosis as a p53-mediated activity during tumour suppression. Nature. 2015;520(7545):57-62.

44. Ou Y, Wang SJ, Li D, et al. Activation of SAT1 engages polyamine metabolism with p53-mediated ferroptotic responses. Proc Natl Acad Sci USA. 2016;113(44):E6806-12.

45. arangelo A, Magtanong L, Bieging-Rolett KT, et al. p53 suppresses metabolic stress-induced ferroptosis in cancer cells. Cell Rep. 2018;22(3):569-75.

46. Xie Y, Zhu S, Song X, et al. The tumor suppressor p53 limits ferroptosis by blocking DPP4 activity. Cell Rep. 2017;15(7):1692-704. 20(.

47. Matsushita K, Mori K, Saritas T, et al. Cilastatin Ameliorates Rhabdomyolysis-Induced Acute Kidney Injury in Mice. J Am Soc Nephrol. 2021. DOI:10.1681/ASN.2020030263.

48. Li WY, Li W, Leng Y, et al. Ferroptosis Is Involved in Diabetes Myocardial Ischemia/Reperfusion Injury Through Endoplasmic Reticulum Stress. DNA Cell Biol. 2020;39(2):210-25.

49. Dixon SJ, Patel DN, Welsch M, et al. Pharmacological inhibition of cystine-glutamate exchange induces endoplasmic reticulum stress and ferroptosis. eLife. 2014;3:e02523.

50. Kamil M, Haque E, Irfan S, et al. ER chaperone GRP78 regulates autophagy by modulation of p53 localization. Front Biosci (Elite Ed). 2017;9:54-66.

51. Guzel E, Arlier S, Guzeloglu-Kayisli O. T et al. Endoplasmic Reticulum Stress and Homeostasis in Reproductive Physiology and Pathology. Int J Mol Sci. 2017;18(4):792.

52. Song M, Cubillos-Ruiz JR. Endoplasmic Reticulum Stress Responses in Intratumoral Immune Cells: Implications for Cancer Immunotherapy. Trends Immunol. 2019;40(2):128-41.

53. Gagliardi M, Cotella D, Santoro C, et al. Aldo-keto reductases protect metastatic melanoma from ER stress-independent ferroptosis. Cell Death Dis. 2019;10(12):902.

54. Lin CC, Ding CC, Sun T, et al. The regulation of ferroptosis by MESH1 through the activation of the integrative stress response. Cell Death Dis. 2021;12(8):727.

55. Qi X, Zhang Y, Guo H, et al. Mechanism and intervention measures of iron side effects on the intestine. Crit Rev Food Sci Nutr. 2020;60(12):2113-25. 
56. Wang N, Zeng G, Yin JL, et al. Artesunate activates the ATF4-CHOP-CHAC1 pathway and affects ferroptosis in Burkitt's Lymphoma. Biochem Biophys Res Commun. 2015;19(3):533-9.

57. Rahmani M, Davis EM, Crabtree TR, et al. The kinase inhibitor sorafenib induces cell death through a process involving induction of endoplasmic reticulum stress. Mol Cell Biol. 2007;27(15):5499-513.

58. Sun X, Ou Z, Xie M, et al. HSPB1 as a novel regulator of ferroptotic cancer cell death. Oncogene. 2015;34(45):5617-25.

59. Yan Z, Liqiong S, Yang YD, et al. Application of multi-dimensional and multi-informational (MD-MI) integrated xanthine oxidase and superoxide anion fingerprint in quality evaluation of Scutellariae Radix. J Pharm Biomed Anal. 2020;191:113595.

60. Lu CL, Liao CH, Lu KC, et al. TRPV1 Hyperfunction Involved in Uremic Toxin Indoxyl Sulfate-Mediated Renal Tubular Damage. Int J Mol Sci. 2020;21(17):E6212.

61. Mi S, Qu Y, Chen X, et al. Radiotherapy Increases 12-LOX and CCL5 Levels in Esophageal Cancer Cells and Promotes Cancer Metastasis via THP-1-Derived Macrophages. Onco Targets Ther. 2020;13:7719-33.

\section{Figures}

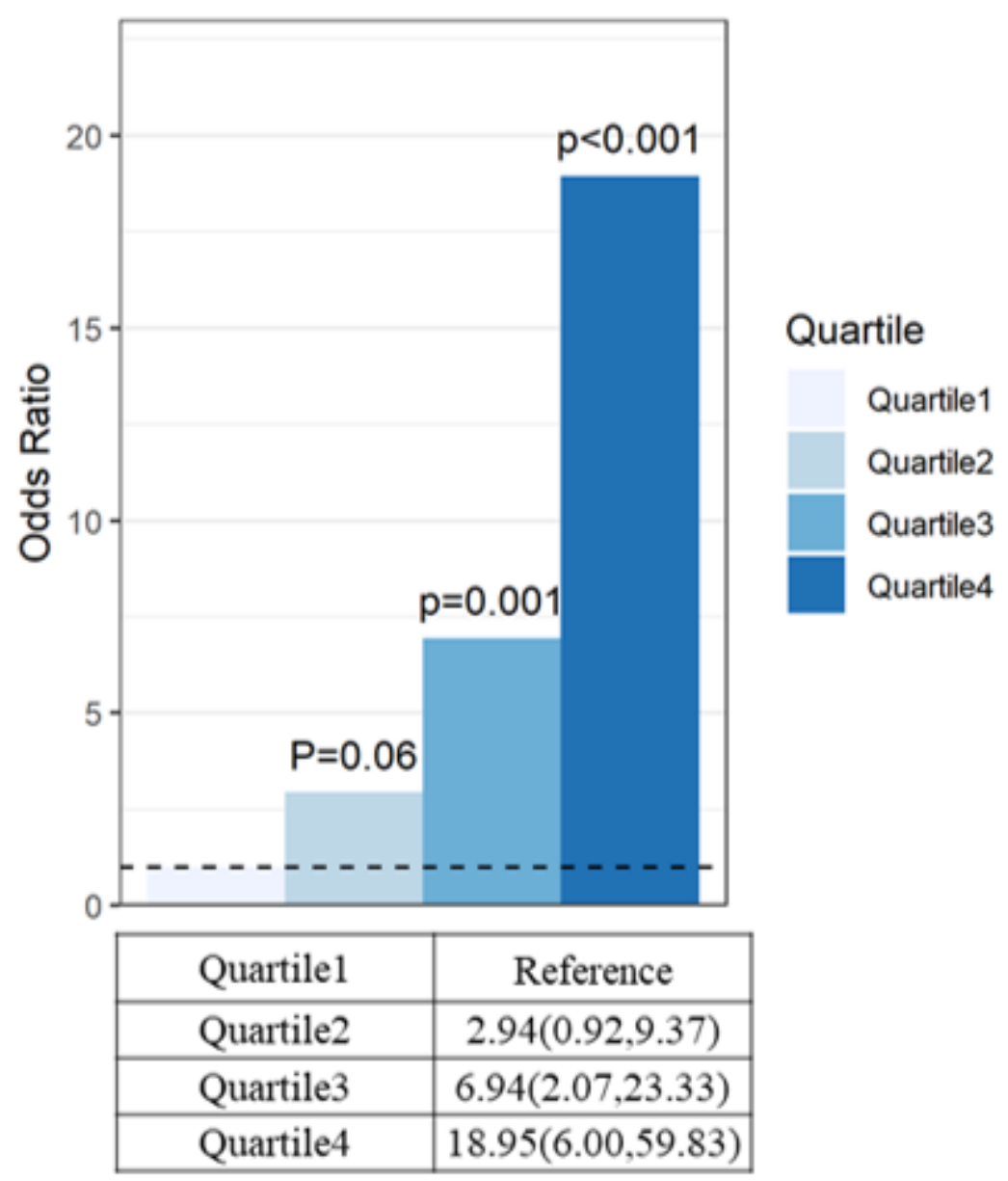


Primary outcome: myoglobin levels and risk of AKI This model was adjusted for age. Quartile 1 was the reference (R) group. Quartile1<127هQuartile2: 127-468.9, Quartile3: 468.9-1000هQuartile4 $\geq 1000$ Quartile1 5/39(12.82\%), Quartile2 12/40(30.0\%), Quartile3 14/28(50.0\%), Quartile4 38/52(73.08\%)

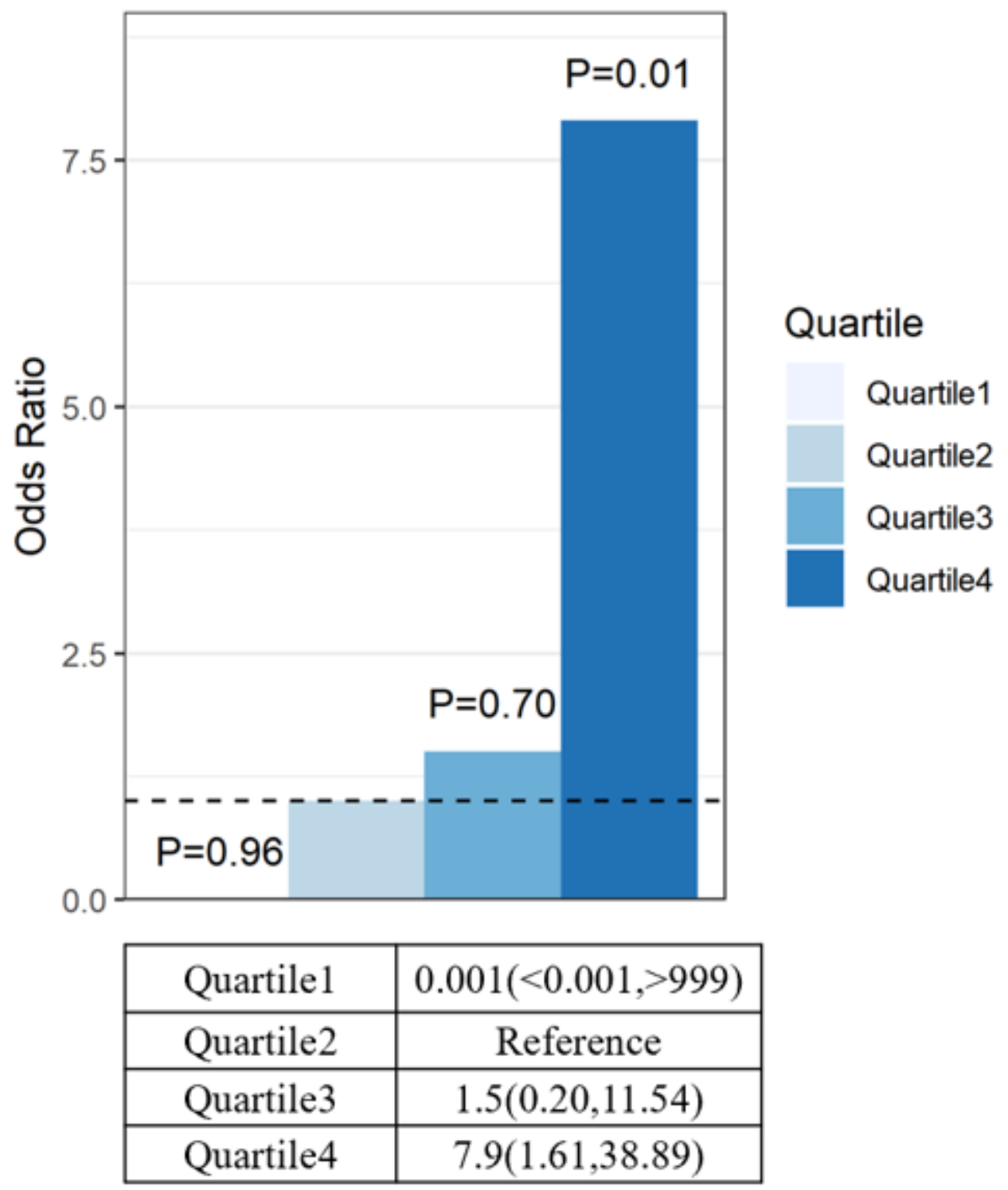

Figure 2

Secondary outcome: myoglobin levels and risk of AKI at discharge and death at 90 days Quartile1 0(0.0\%), Quartile2 3/40(7.5\%), Quartile3 3/29(10.3\%), Quartile4 18/52(34.6\%) 
A

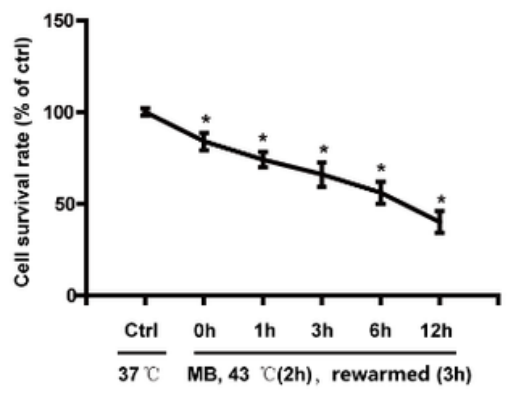

C

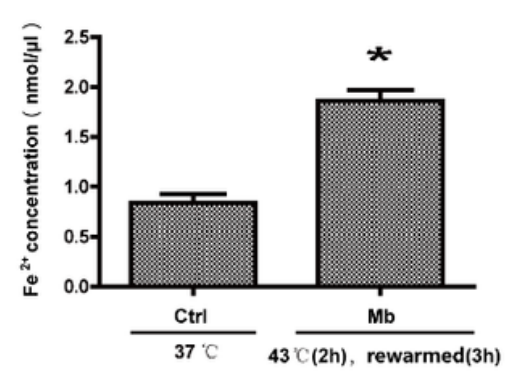

B

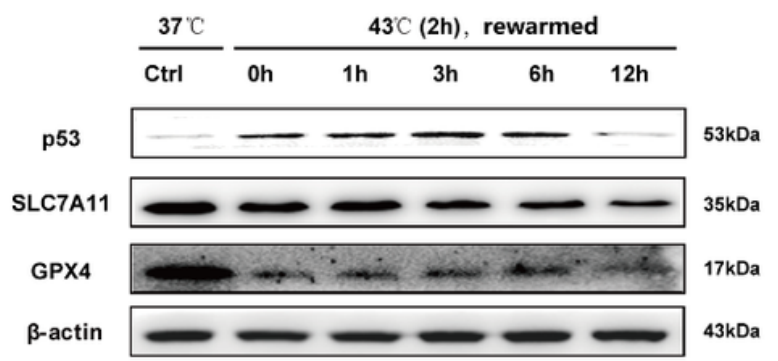

D

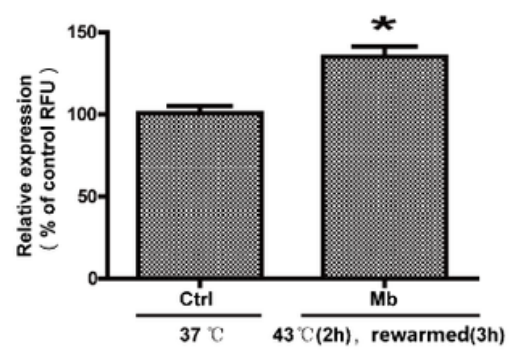

B1

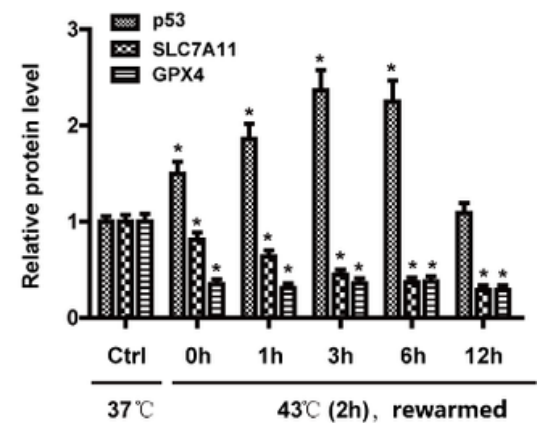

E

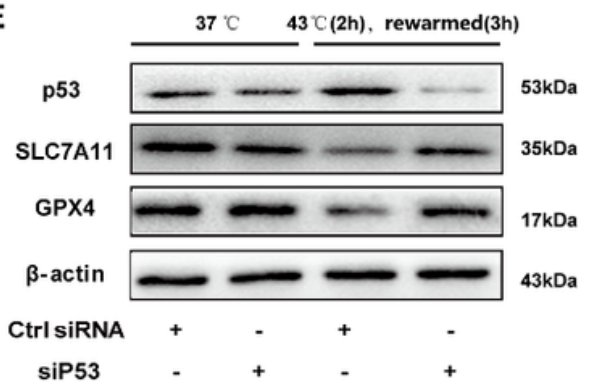

E1

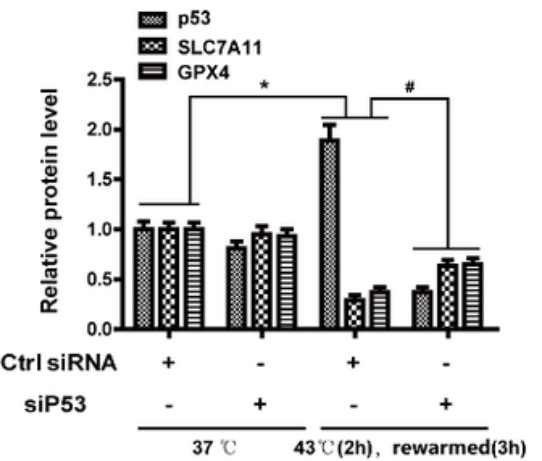

Figure 3

Myoglobin is Associated with HK-2 Cell Ferroptosis in a Heat Stress Model. (A) Human HK-2 cells were treated with myoglobin at concentrations of $882.35 \mathrm{~nm} / \mathrm{l}$ at $43^{\circ} \mathrm{C}$ for 2 hours and rewarmed at $37^{\circ} \mathrm{C}$ at different time points $(0 \mathrm{~h}, 1 \mathrm{~h}, 3 \mathrm{~h}, 6 \mathrm{~h}$, and $12 \mathrm{~h})$. The effect of heat stress and myoglobin on the survival of HK-2 cells and the cell survival rate were examined. (B) Western blot and quantitative analyses (B1) were performed to evaluate the expression of P53, SLC7A11 and GPX4. (C and D) Intracellular Fe2+ and ROS in HK-2 cells were detected. (E, E1) HK-2 cells were transfected with control (ctrl) siRNA or siP53 for $48 \mathrm{~h}$, treated with myoglobin at a concentration of $882.35 \mathrm{~nm} / \mathrm{I}$ at $43^{\circ} \mathrm{C}$ for 2 hours, and rewarmed at 37 ${ }^{\circ} \mathrm{C}$ for $3 \mathrm{~h}$. Western blot and quantitative analyses were performed evaluate the expression of P53, SLC7A11 and GPX4. The values are the mean \pm S.D. from triplicate independent experiments with statistical significance: ${ }^{*} P<0.05$ vs. the control group, $\# p<0.05$ vs. the scrambled+ rewarmed group. 
ATF-4

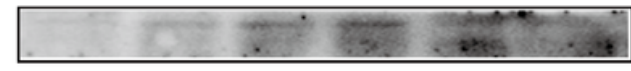

ATF-6

p-IRE1

IRE1
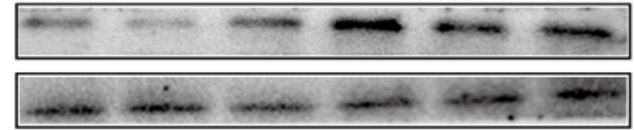

CHOP

$\beta$-actin

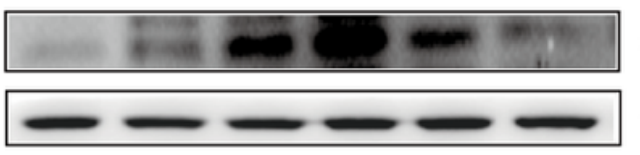

B

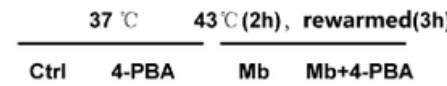

p-PERK

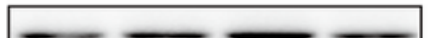

PERK

p-elF2 $\alpha$

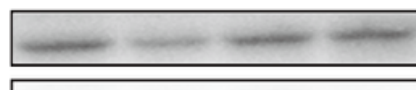

$170 \mathrm{kDa}$

$140 \mathrm{kDa}$

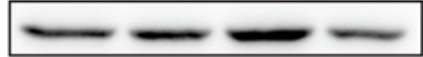

$38 \mathrm{kDa}$

elF2 $\alpha$

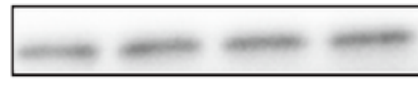

ATF- 4

ATF-6

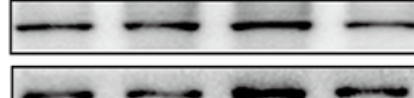

$38 \mathrm{kDa}$

$49 \mathrm{kDa}$

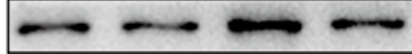

$75 \mathrm{kDa}$

p-IRE1

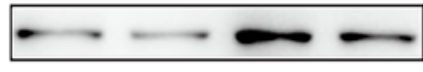

IRE1

CHOP

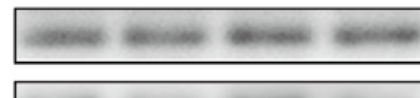

$\beta$-actin

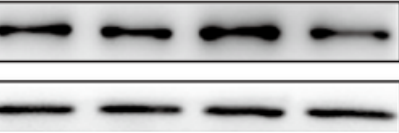

$49 \mathrm{kDa}$

$75 \mathrm{kDa}$

$110 \mathrm{kDa}$

$110 \mathrm{kDa}$

$27 \mathrm{kDa}$

$43 \mathrm{kDa}$

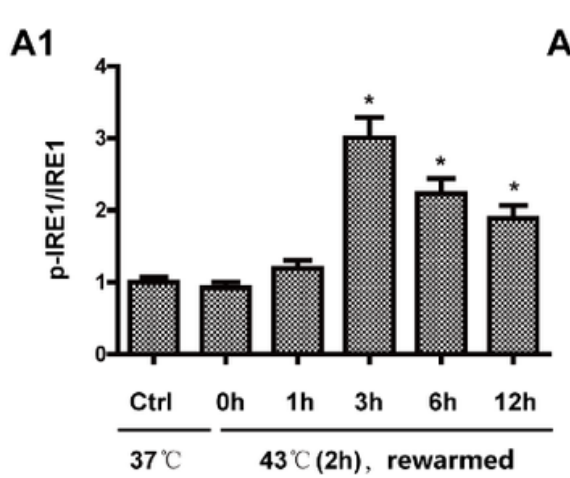

A2

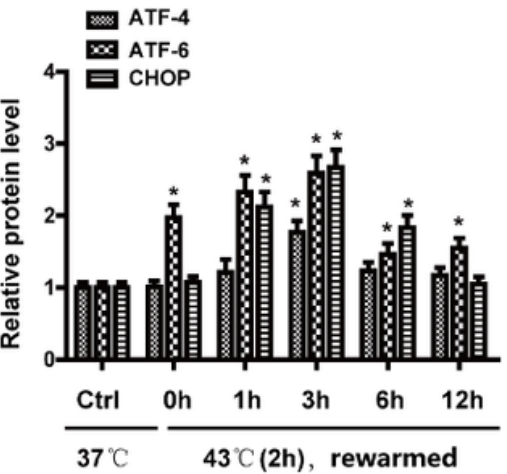

B1

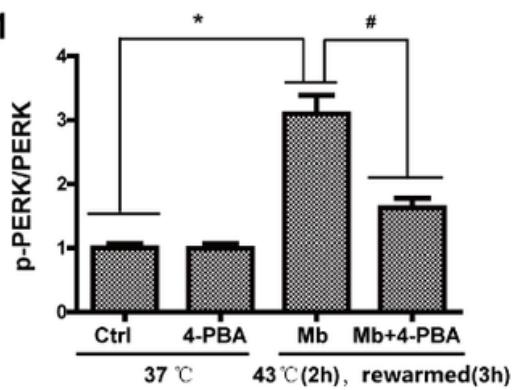

B2

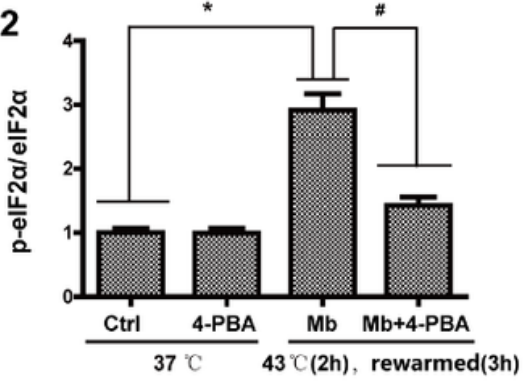

B3

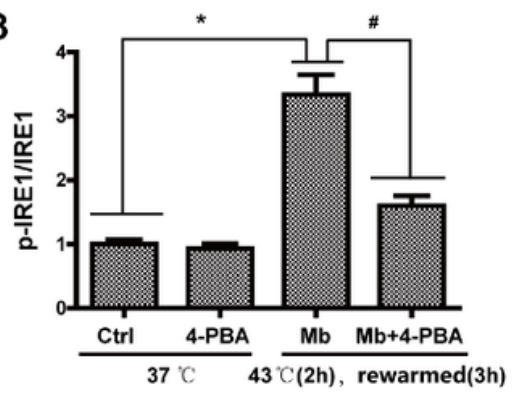

B4

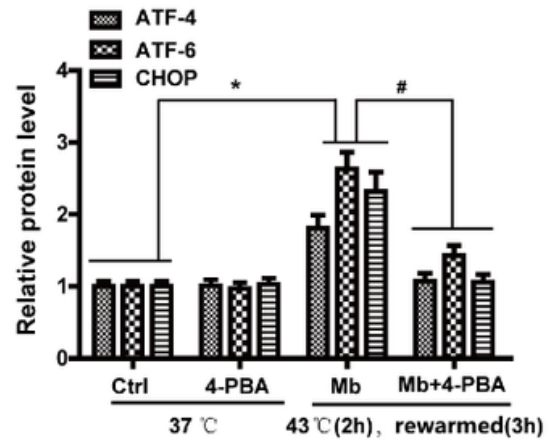

Figure 4

Myoglobin aggravates the endoplasmic reticulum stress response of HK-2 cells after heat stress. (A, A1) Human HK-2 cells were treated with myoglobin at concentrations of $882.35 \mathrm{~nm} / \mathrm{l}$ at $43{ }^{\circ} \mathrm{C}$ for 2 hours and rewarmed at $37^{\circ} \mathrm{C}$ at different time points $(0 \mathrm{~h}, 1 \mathrm{~h}, 3 \mathrm{~h}, 6 \mathrm{~h}$, and $12 \mathrm{~h})$. The expression levels of $\mathrm{p}$ IRE1/IRE1, ATF-4, ATF-6 and CHOP were measured by Western blot. (B, B1, B2, B3, B4) Human HK-2 cells were pretreated with 4-BPA, treated with myoglobin at concentrations of $882.35 \mathrm{~nm} / \mathrm{I}$ at $43{ }^{\circ} \mathrm{C}$ for 2 hours, and rewarmed at $37^{\circ} \mathrm{C}$ for $3 \mathrm{~h}$. The expression levels of p-PER/PERK, p-elF2, p-IRE1/IRE1, ATF-4, ATF-6 and CHOP were measured by Western blot. The values are the mean \pm S.D. from triplicate independent experiments with statistical significance: ${ }^{\star} \mathrm{P}<0.05$ vs. the control group, $\# p<0.05$ vs. the Mb group. 
A

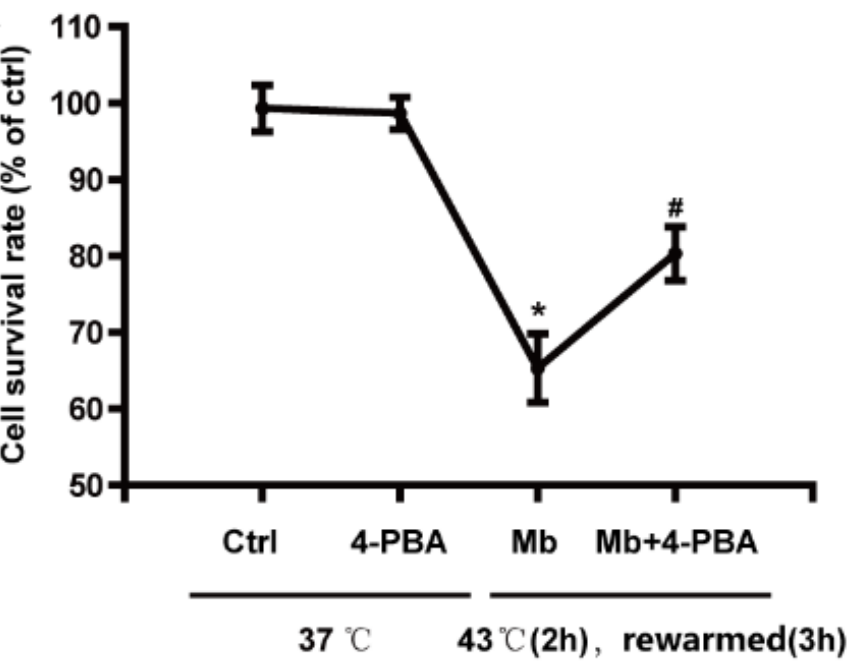

B1

B
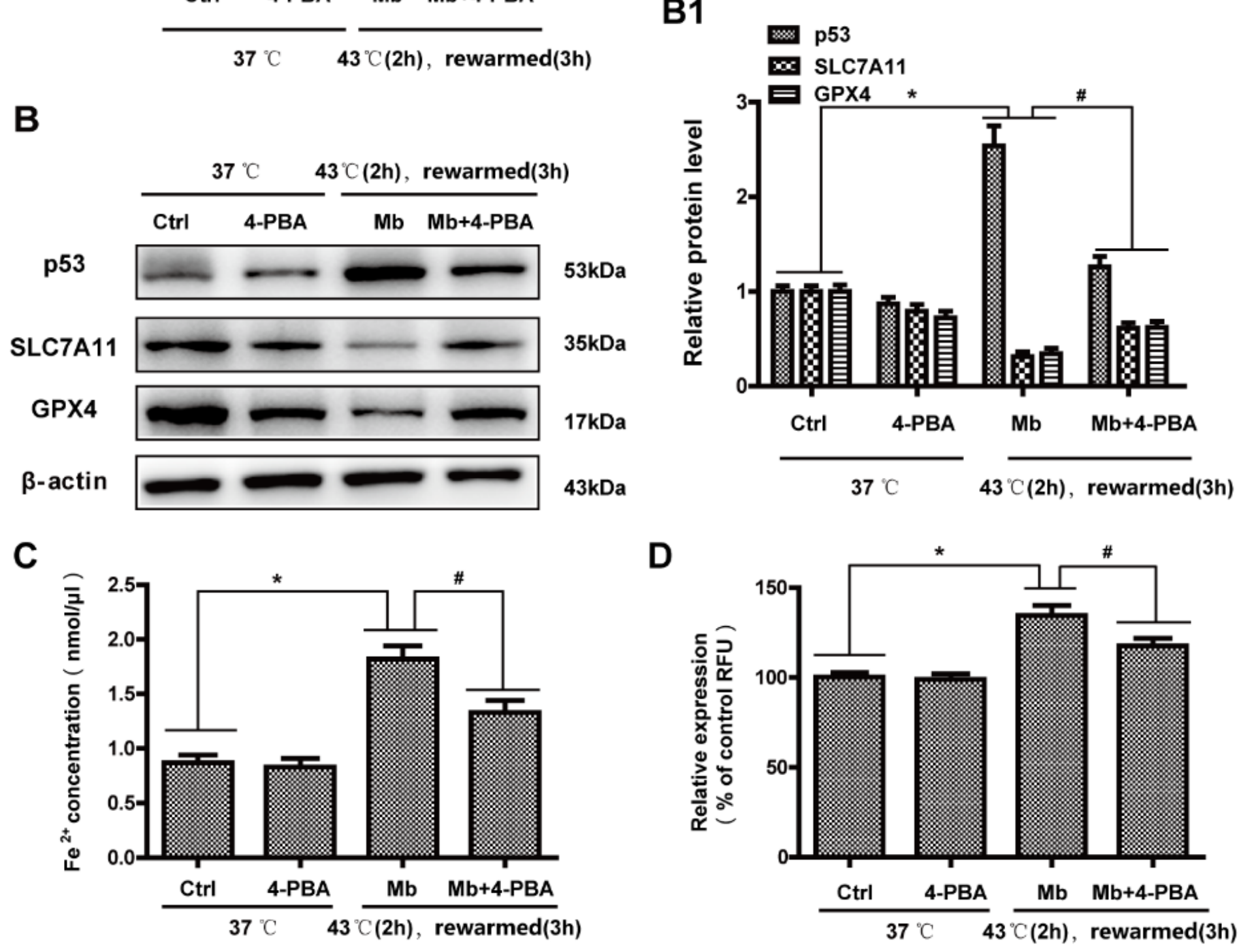

D

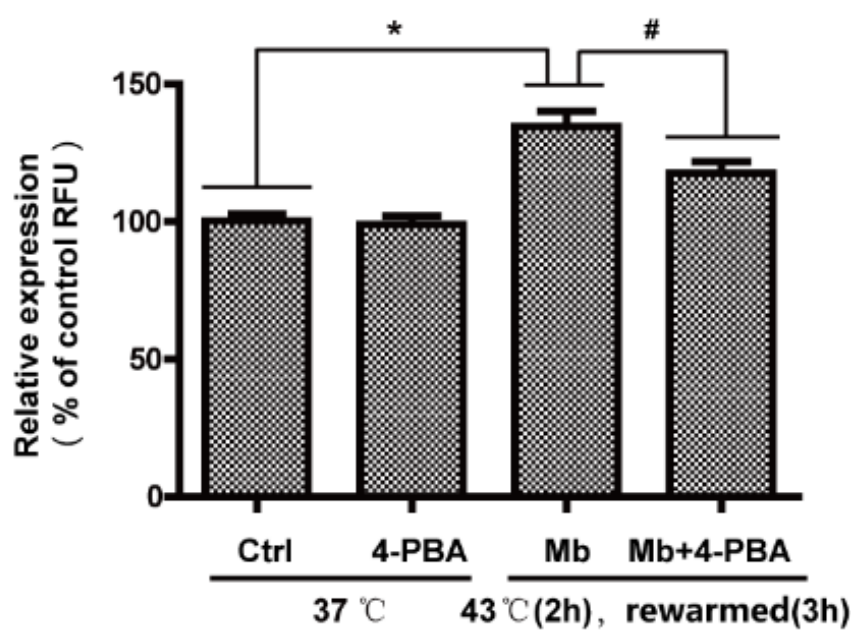

Figure 5

Myoglobin modulates HK-2 cell Ferroptosis involving Endoplasmic Reticulum Stress after Heat Stress. (A) Human HK-2 cells were pretreated with 4-BPA, treated with myoglobin at concentrations of $882.35 \mathrm{~nm} / \mathrm{l}$ at $43^{\circ} \mathrm{C}$ for 2 hours, and rewarmed at $37^{\circ} \mathrm{C}$ for $3 \mathrm{~h}$. The cell survival rate of HK-2 cells was examined by CCK8. (B) Western blot and quantitative analyses (B1) were performed to evaluate the expression of P53, SLC7A11 and GPX4. (C and D) Intracellular Fe2+ and ROS in HK-2 cells were detected. The values are the 
mean \pm S.D. from triplicate independent experiments with statistical significance: ${ }^{*}<0.05$ the control group, $\# p<0.05$ vs. the Mb group.
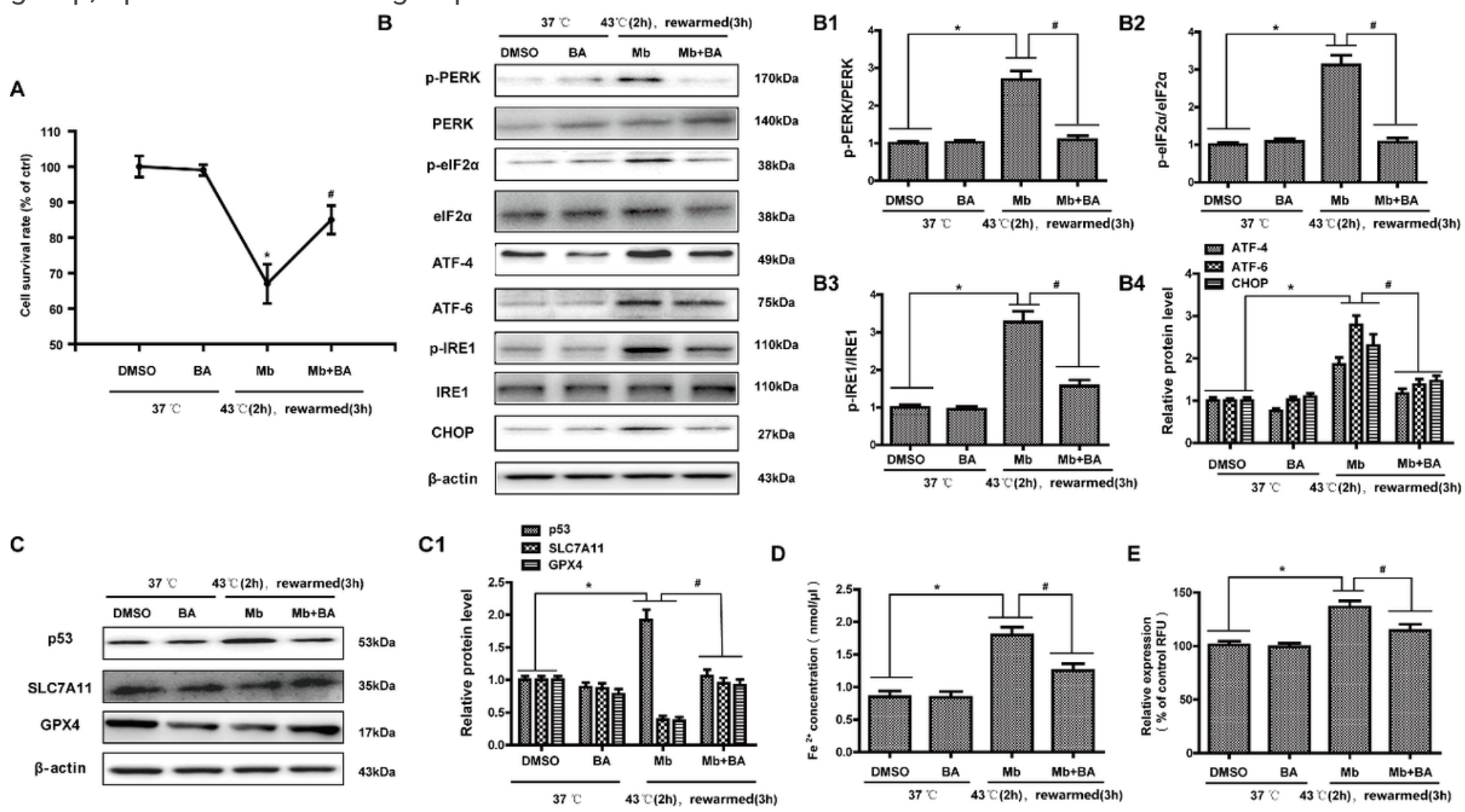

Figure 6

Baicalein Protects HK-2 Cell Ferroptosis Induced by Myoglobin under Heat Stress by Inhibiting Endoplasmic Reticulum Stress. (A) Human HK-2 cells were treated with myoglobin at a concentration of $882.35 \mathrm{~nm} / \mathrm{I}$ and baicalein (Selleck Chemicals, USA) at a concentration of $20 \mu \mathrm{M}$ at $43^{\circ} \mathrm{C}$ for 2 hours and rewarmed at $37{ }^{\circ} \mathrm{C}$ for 3 hours. HK-2 cells were treated with DMSO as control. The cell survival rate of HK2 cells was examined by CCK8. (B, B1, B2, B3, B4) Total protein was stained, and p-PER/PERK, p-elF2, pIRE1/IRE1, ATF-4, ATF-6 and CHOP were analysed by Western blotting. (C) Western blot and quantitative analyses (C1) were performed to evaluate the expression of P53, SLC7A11 and GPX4. (D and E) Intracellular Fe2+ and ROS in HK-2 cells were detected. The values are the mean \pm S.D. from triplicate independent experiments with statistical significance: ${ }^{*} P<0.05$ the DMSO group, $\# p<0.05$ vs. the Mb group.

\section{Supplementary Files}

This is a list of supplementary files associated with this preprint. Click to download.

- GraphicalAbstract.png 